\title{
Acute Myeloid Leukemia Presenting as a Cardiac Mass
}

\author{
Alia Magzoub Mohamed ${ }^{\mathrm{a}}$ Maysa Saleh ${ }^{\mathrm{a}}$ Fatima Alzarawani $^{\mathrm{a}}$ \\ Nermeen El-kholy ${ }^{b}$ \\ aDepartment of Pediatrics, Latifa Women and Children Hospital, Dubai, UAE; 'bepartment of Pediatric Cardiology, \\ Al Jalila Children's Specialty Hospital, Dubai, UAE
}

\section{Keywords}

Cardiac · Cardiac mass - Acute myeloid leukemia - Myeloid sarcoma $\cdot$ Oncology $\cdot$ Pediatrics

\begin{abstract}
Leukemias are the most common childhood malignancy. It is a systemic disease presenting with different signs and symptoms. Extramedullary manifestations, in particular myeloid sarcoma (MS), are extremely rare, often challenging to identify, even more challenging to diagnose, and more than often misdiagnosed owing to its similarity with other solid tumors. We describe a previously healthy boy, aged 4 years, who had a cardiac mass lesion, presumed to be a MS, noted in the right atrium at the time of initial routine echocardiography. The lesion resolved without surgical intervention following the institution of multi-agent chemotherapy.
\end{abstract}

(c) 2020 The Author(s)

Published by S. Karger AG, Basel

\section{Introduction}

Acute leukemia is the most common malignancy in children and accounts for approximately $30 \%$ of all childhood malignancies $[1,2]$. It can rarely present as an extramedullary tumor mass (known as myeloid sarcoma
(MS)) consisting of myeloid blasts which efface the tissue architecture [3]. MS can develop in any area of the body, including the cervix, mediastinum, small intestines, lymph nodes, and skin [4].

It has been reported that MS occurs in $0.8-9 \%$ of patients with myelogenous leukemia [5-7] especially in situations of cytogenetic abnormalities such as $t(8 ; 22)$, inv(16), and 11q23 [8]. MS can occur following, concurrently with, or preceding bone marrow involvement of AML. The presence of MS adversely affected the relapse and disease-free survival rates in adult patients with AML. The present study reports a case of MS derived from the right atrium (RA) and reviews the associated literature, in order to improve the understanding of the disease and provide a reference for standardized and individualized treatments.

\section{Case Report}

A boy aged 4 years was admitted to our hospital with a history of fever, abdominal pain, chest pain, and breathing difficulty of 5 days duration. There was no past medical history of note although his parents had a history of allergies, but his only sibling was healthy. Clinical examination was unremarkable. On admission, he was febrile and mildly tachypneic. He was alert and orientated.
C 2020 The Author(s)

Published by S. Karger AG, Basel

This article is licensed under the Creative Commons AttributionNonCommercial-NoDerivatives 4.0 International License (CC BY NC-ND) (http://www.karger.com/Services/OpenAccessLicense) Usage and distribution for commercial purposes as well as any distribution of modified material requires written permission.
Alia Magzoub Mohamed Department of Pediatrics Latifa Women and Children Hospital

Oud Maitha Street, PO Box-9115, Dubai (United Arab Emirates) amagzoub@dha.gov.ae 
Table 1. Initial CBC on admission

\begin{tabular}{lcr}
\hline WBC count & $5.0-15.0 \times 10^{3} / \mu \mathrm{L}$ & $\mathbf{9}$ \\
Hemoglobin, blood & $11.0-14.0 \mathrm{~g} / \mathrm{dL}$ & $\mathbf{1 2 . 2}$ \\
Platelets count & $200-490 \times 10^{3} / \mu \mathrm{L}$ & $\mathbf{3 4 0}$ \\
Neutrophil, \% & $\%$ & 54.4 \\
Lymphocyte, \% & $\%$ & 36.8 \\
Eosinophil, \% & $\%$ & 0.4 \\
Monocyte, \% & $\%$ & 7.9 \\
\hline
\end{tabular}

CBC, complete blood count; WBC, white blood cell.

Table 2. CBC before the initial BMA

\begin{tabular}{lcr}
\hline WBC count & $5.0-15.0 \times 10^{3} / \mu \mathrm{L}$ & $\mathbf{2 5 . 9}$ \\
Hemoglobin, blood & $11.0-14.0 \mathrm{~g} / \mathrm{dL}$ & $\mathbf{9 . 7}$ \\
Platelets count & $200-490 \times 10^{3} / \mu \mathrm{L}$ & $\mathbf{8 7}$ \\
Neutrophil, \% & $\%$ & 27.7 \\
Lymphocyte, \% & $\%$ & 62.9 \\
Eosinophil, \% & $\%$ & 0.2 \\
Monocyte, \% & $\%$ & 8.4 \\
\hline
\end{tabular}

CBC, complete blood count; BMA, bone marrow aspirate.

Table 3. Shows the latest CBC post-chemotherapy

\begin{tabular}{lcr}
\hline WBC count & $5.0-15.0 \times 10^{3} / \mu \mathrm{L}$ & $\mathbf{1 0 . 6}$ \\
Hemoglobin, blood & $11.0-14.0 \mathrm{~g} / \mathrm{dL}$ & $\mathbf{1 4}$ \\
Platelets count & $200-490 \times 10^{3} / \mu \mathrm{L}$ & $\mathbf{2 8 7}$ \\
Neutrophil, \% & $\%$ & 50 \\
Lymphocyte, \% & $\%$ & 46 \\
Eosinophil, \% & $\%$ & 0.04 \\
Monocyte, \% & $\%$ & 0.00 \\
\hline
\end{tabular}

CBC, complete blood count; WBC, white blood cell.

Vital signs were BP 120/80 mm Hg, pulse $118 \mathrm{bpm}$, temperature $38.6^{\circ} \mathrm{C}$, respiratory rate $26 / \mathrm{min}$, and $\mathrm{SpO} 294 \%$ on room air. Growth parameters were height 90th centile and weight 92nd centile. Respiratory examinations demonstrated decreased air entry on the right side with crackles. Cardiovascular examination revealed normal heart sounds with a soft systolic murmur.

Abdominal examination revealed soft nontender abdomen with mild hepatomegaly with the liver edge palpable $3-4 \mathrm{~cm}$ below the costal margin. There was no splenomegaly, however.

The initial CBC (Table 1) initial chest X-ray (Fig. 1) showed right perihilar and right lower zone opacity silhouetted heart border. Pneumonic consolidation blunted right cost-phrenic sinus (mild pleural effusion). Chest ultrasound showed bilateral lower lobes consolidation with bilateral pleural effusion - more on the right side.

The patient was started on intravenous (IV) fluids, IV ceftriaxone, and IV vancomycin in addition to nebulized bronchodilators

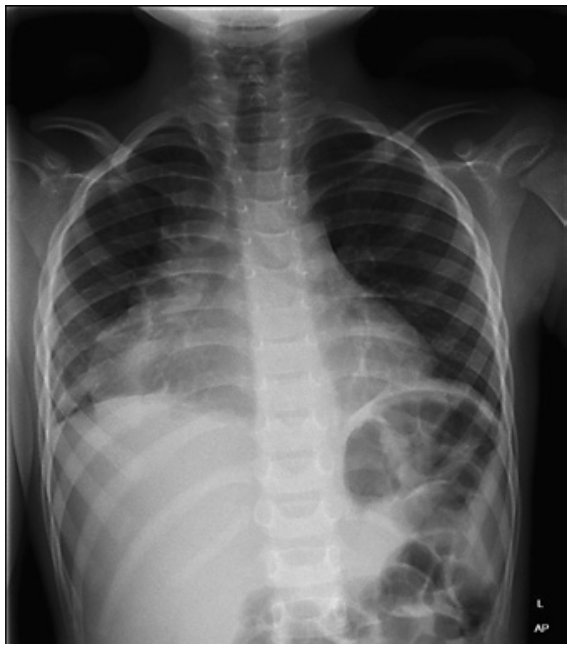

Fig. 1. Chest X-ray showing right perihilar and right lower zone opacity silhouetting heart border. Pneumonic consolidation, blunted right costophrenic sinus with mild pleural effusion.

as the sputum was positive for mycoplasma. Oral azithromycin was added.

On day 5, chest ultrasound was repeated and showed progressive bilateral pleural effusion. Abdominal ultrasound showed mild ascites. On day 6 , the patient developed a puffy face with bilateral lower limb swelling, hence, IV fluids were restricted and kept on neutral fluid balance and the electrolytes were corrected accordingly.

The liver function tests were deranged with low albumin and decreased fibrinogen level. Hence, albumin was transfused.

Coagulopathy was noted with increased prothrombin time and low platelets; hence, Vit $\mathrm{K}$ was given in addition to fresh frozen plasma, cryoprecipitates as supportive measurement. IV ceftriaxone was changed to IV meropenem, and IV vancomycin was discontinued.

On day 7, an echocardiogram showed a mass originating from the free wall of the RA invading the wall and extending outside. The intracardiac mass was large and protruding through the tricuspid valve and obstructing it into the right ventricle. In addition, the left ventricular systolic function was found to be poor (see online suppl. video 1 at www.karger.com/doi/10.1159/000511162).

A computed tomography (CT) angiography of the chest and abdomen with contrast showed large ill-defined solid hypodense right intracardiac and extracardiac mass invading the anterior atrial wall, the mass had lobulated outlines and measured about $5.4 \mathrm{~cm}$ AP-5.7 cm transversely and $6.5 \mathrm{~cm}$ craniocaudal, the mass was engulfing the right coronary artery with no gross invasion or stenosis. The mass is not calcified, nor necrotic. There was mild to moderate pericardial effusion and multiple mediastinal enlarged lymph nodes were noted bilateral small-volume pleural effusion and moderate ascites (Fig. 2a, b). Daily echocardiograms consistently showed the same results. A bone marrow examination was planned, but the parents opted to continue further treatment in India. $\mathrm{CBC}$, before the initial bone marrow examination, is shown in Table 2. The initial bone marrow aspirate (BMA) showed as AML M4/M5, flow cytometry confirmed AML M4 morphology, 

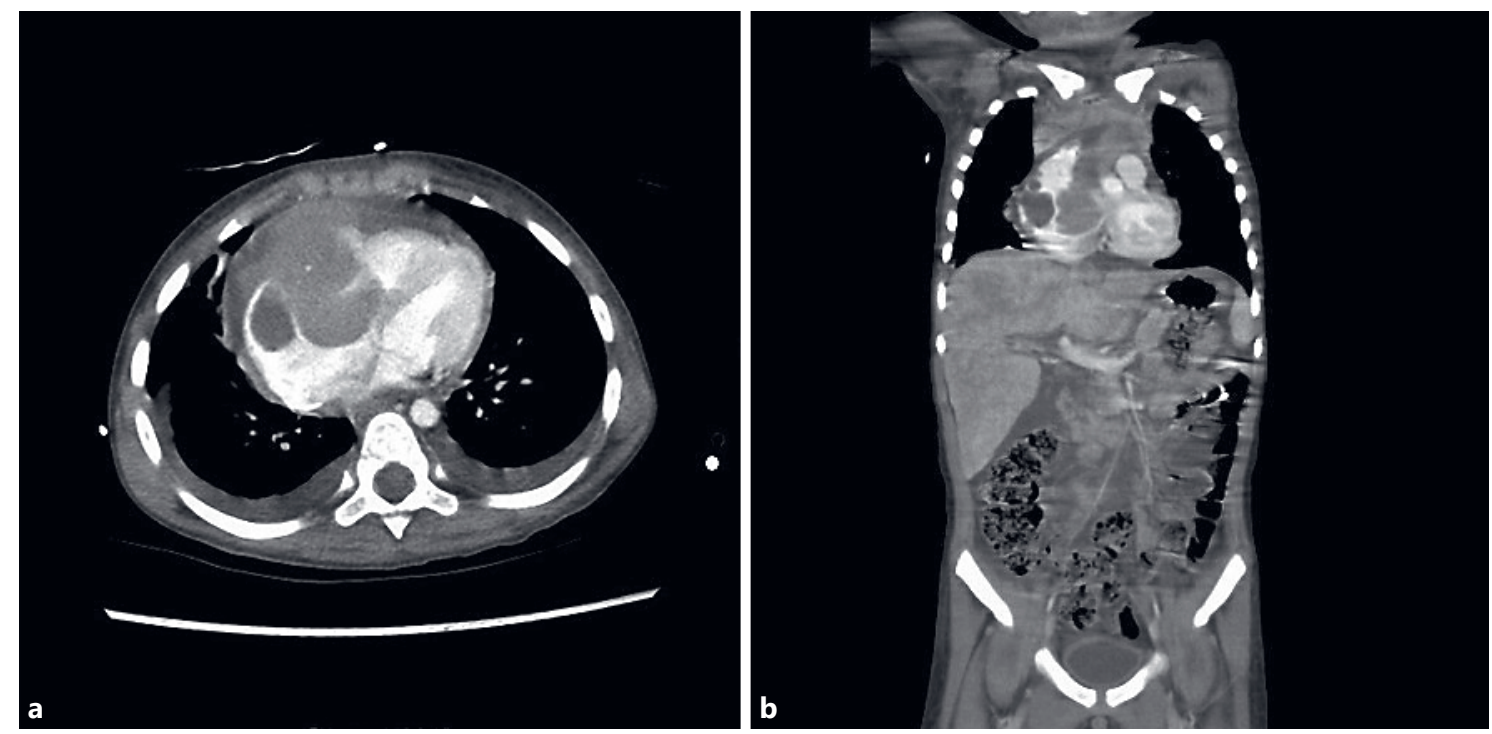

Fig. 2. a CT of the chest with contrast showing a large ill-defined, lobulated solid mass epicentered within the right atrium, invading the anterior atrial wall and connected to a right anterolateral extra cardiac component. $\mathbf{b}$ CT of the chest-abdomen-pelvis with contrast showing an anterior superior pericardial breach. The mass is engulfing the right coronary artery with no gross invasion or stenosis.
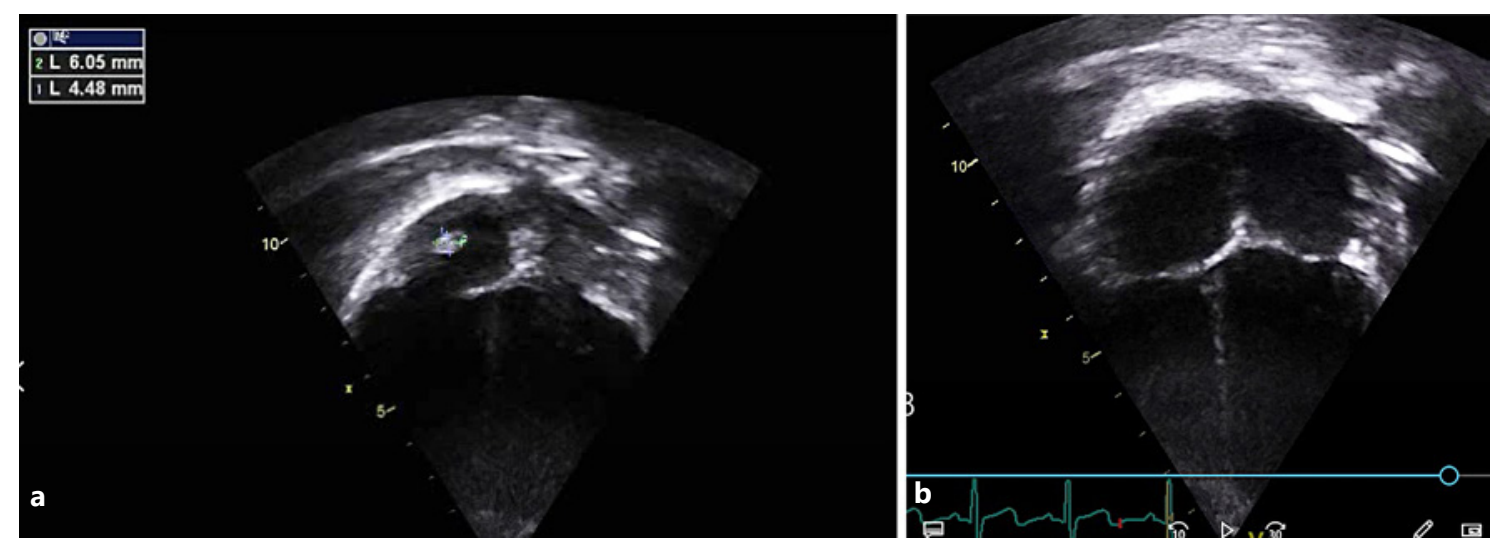

Fig. 3. a, b Echocardiology showing resolution of the intracardiac mass after chemotherapy treatment.

molecular markers, and cytogenetic showed FISH inv 16 positive, FLT3 D853 mutation positive, and FLT 3 ITD negative (molecular markers).

According to the result of the bone marrow test, the probability that cardiac mass was an MS resulting from AML was at the helm of differential diagnosis proposed. At this stage, due to the patient's poor condition of respiratory distress, severe thrombocytopenia, and coagulopathy, it was not possible to perform cardiac lesions' biopsy, and the patient was treated with induction chemotherapy with 2 cycles of induction. The first cycle was standard cytarabine and etoposide (daunorubicin was deferred in view of cardiac involvement). Follow-up BMA was in morphological remission. Subsequent echo demonstrated $50 \%$ reduction in the cardiac mass,
MRD $0.9 \%$. The second cycle was etoposide, cytarabine, and daunorubicin. Follow-up BMA revealed morphological remission.2.

Repeat echo showed significant reduction in the cardiac mass size to $1-2 \mathrm{~cm}, \mathrm{MRD}$ was $<0.1 \%$. Two consolidation cycles of $\mathrm{Hi}$ $\mathrm{DAC}$ were given repeat bone marrow examination showed morphological remission MRD $<0.47 \%$. Follow-up echo revealed only 1-cm hyperdense residual cardiac mass lesion (Fig. 3a, b). Parents were counseled for hematopoietic stem cell transplant regarding the risk of relapse in view of extramedullary disease at onset and the persistent positive MRD.

The treating team decided for clinical follow-up planned for BMA and MRD 3 months later or earlier if required, fortunately his BMA report showed morphological remission, with MRD $<0.1 \%$. Latest FBC is shown in Table 3 . 


\section{Discussion}

In the pediatric population, cardiac mass, whether primary or secondary, is extremely rare. The incidence of cardiac tumors in children is up to $0.08 \%$ in autopsy series. Though primary cardiac tumors are usually benign, $10 \%$ were found to be malignant. The prevalence of secondary malignant tumors is $10-20$ times than primary ones. It is most commonly caused by melanoma, lymphoma, leukemia, and carcinoma of the lung, breast, and esophagus. Interestingly, over $90 \%$ of secondary cardiac tumors remain asymptomatic [9-12].

Cardiac complications of the pediatric patients with acute leukemia are common. Most of the cardiac complications may be due to chemotherapeutics such as antracyclins, besides anemia, infections, or direct leukemic infiltrations of the heart or MS.

Because of the lack of diagnostics algorithm, recognition of cardiac MS is a real diagnostic challenge, as a broad panel of diagnostic tools has to be used. The diagnostic approach must include different imaging studies, such as cardiac echocardiography, CT, and CMR [13], and histological, immunohistochemical, and genetic analysis of blood, bone marrow, and, if possible, tumor tissue. The best diagnostic approach to recognizing MS is a biopsy of the tumor and immunohistochemical examination. However, the standard immunohistochemical panel in diagnosing MS is lacking which makes it difficult to distinguish MS from other neoplasms, in particular nonHodgkin lymphomas $[14,15]$. In our case, the patient was coagulopathic and thrombocytopenic; hence, biopsy was not possible, and he was too restless and sick for CMR, so echo and cardiac CT were performed together with the histological evaluation of bone marrow.

The therapeutic algorithm for MS is unclear in children and adults. Prospective trials are lacking; therefore, treatment recommendations are based on retrospective studies which focus on adult patients. Available treatment options include systemic chemotherapy, surgical resection, radiotherapy, hematopoietic stem cell transplantation (HSCT), or a combination of these methods. In MS associated with systemic disease, AML chemotherapy is also recommended, with consideration of alloHSCT, especially if other unfavorable prognostic factors are present (age, cytogenetics) [9].

Our patient was treated as an AML with an extramedullary leukemia presentation presumably (MS), following the institution of multi-agent chemotherapy. On followup, there was regression of the cardiac mass and bone marrow morphological clearance. The cardiac mass al- most resolved without surgical intervention. MS is a rare neoplasm whose knowledge is mostly based on case reports and/or technically dated contributions [2].

MS (also termed extramedullary AML, extramedullary myeloid tumor, and granulocytic sarcoma) is a rare manifestation of AML characterized by the occurrence of 1 or more myeloid tumor masses (or infiltrations) at an extramedullary site. It can occur almost anywhere in the body and can occur at any time over the course of the disease, de novo, or as relapse $[4-9,13-18]$. It may be difficult to diagnose because of its similarity to other neoplasms [2, $3,5]$. MS is most frequent in young children (median age, 5 years), and its incidence decreases with age.

In the literature, the incidence rate in children varies, but it may be significantly higher in patients with AML. Patients with extramedullary presentation of the disease were more frequently diagnosed with types M4 and M5 leukemia [5]. The presence of MS at diagnosis of pediatric AML is associated with poor clinical outcomes [7].

They are most common in children and may present at any time during the course of the disease, concurrently with the onset of leukemia, or during a remission or relapse. They occasionally precede the clinical onset of $A M L$, presenting a diagnostic challenge $[3,5,7]$. In the literature, the incidence rate in children with AML varies may reach $40 \%$, but it may be significantly higher in patients with AML, and was more frequently diagnosed with types M4 and M5 leukemia, with higher initial white blood cell count $[5,7]$.

Because of its different localization and symptoms, and the lack of diagnostics algorithm, MS is a real diagnostic challenge, recognition of MS is more challenging, as a broad panel of diagnostic tools has to be used. The diagnostic approach must include different imaging studies, histological, immunohistochemical, and genetic analysis of blood, bone marrow, and, if possible, tumor tissue. The best diagnostic approach to recognizing MS is a biopsy of the tumor and immunohistochemical examination. However, the standard immunohistochemical panel in diagnosing MS is lacking which makes it difficult to distinguish MS from other neoplasms, in particular nonHodgkin lymphomas [5, 7].

The correct diagnosis of MS is important for adequate therapy, which is often delayed because of a high misdiagnosis rate. The therapeutic algorithm for MS is unclear in children and adults. Prospective trials are lacking; therefore, treatment recommendations are based on retrospective studies which focus on adult patients. Available treatment options include systemic chemotherapy, surgical resection, radiotherapy, HSCT, or a combination 
of these methods. In MS associated with systemic disease, AML chemotherapy is also recommended, with consideration of alloHSCT, especially if other unfavorable prognostic factors are present (age, cytogenetics) [5].

Herein, we are reporting a previously healthy boy who was admitted with tachypnea and fever. His ECHO and CT scan with contrast showed an intracardiac mass with extracardiac extension. The possibilities include rhabdomyosarcoma, angiosarcoma, lymphoma of pericardium/ cardiac, and less likely teratoma. Hence, thorough investigation was carried out to rule out every possible etiology, primary and secondary causes.

In the pediatric population, cardiac mass, whether primary or secondary, is extremely rare. The incidence of cardiac tumors in children is up to $0.08 \%$ in autopsy series. Though primary cardiac tumors are usually benign, $10 \%$ were found to be malignant. The prevalence of secondary malignant tumors is $10-20$ times than primary ones. It is most commonly caused by melanoma, lymphoma, leukemia, and carcinoma of the lung, breast, and esophagus. Interestingly, over than $90 \%$ of secondary cardiac tumors remain asymptomatic $[9-11,15]$.

Cardiac complications of the pediatric patients with acute leukemia are common. Most of the cardiac complications may be due to chemotherapeutics such as antracyclins, besides anemia, infections, or direct leukemic infiltrations of the heart.

Regarding intracardiac mass, echocardiography, CT, and MRI of the heart are the main noninvasive diagnostic tools. Open surgical or endomyocardial biopsy is only utilized to reveal the histology of the lesion before surgical resection. A thorough metastatic check should be carried out with CT or MRI imaging before removal of malignant cardiac tumors [5]; in our case, there was regression of the mass post-chemotherapy.

Cardiac CT can provide useful anatomic and functional information as an adjunct to echocardiography and MR in the evaluation of cardiac masses. With high spatial and contrast resolution, fast acquisition times, and the capability to identify calcification and fat, cardiac CT can serve as an ideal alternative to MR imaging, especially in patients with contraindications. Although cardiac MR imaging is the preferred modality, CT can also play an important role in the evaluation of cardiac masses. Not only can CT provide anatomic information and tissue characterization such as calcification, but this modality offers more comprehensive assessment of patients with suspected metastatic disease. So, cardiac CT can serve as an ideal alternative to MR imaging, especially in patients with contraindications $[9-11,15]$.

A Cardiac Mass as an Initial Presentation of AML in Children
The therapeutic algorithm for MS is unclear in children and adults. In MS associated with systemic disease, AML chemotherapy is also recommended, with consideration of alloHSCT, especially if other unfavorable prognostic factors are present (age and cytogenetics). The role of alloHSCT is highlighted in several studies, in which it has been shown that alloHSCT improves the overall survival of patients with MS [5]. However, pediatric patients with AML and MS may not benefit from SCT [6].

Our patient was treated as an AML with an extramedullary leukemia presentation presumably (MS). Following the institution of multi-agent chemotherapy includes standard daunorubicin and cytarabine induction therapy for MS [5].

On follow-up, there was regression of the cardiac mass and bone marrow morphological clearance. The cardiac mass almost resolved without surgical intervention.

\section{Conclusion}

We report the case of AML associated with an invasive cardiac mass that presented with nonspecific symptoms. MS is not well known, and many patients with the disease have been assigned an incorrect diagnosis at their first evaluation. Although it is difficult to confirm the diagnosis - especially when MS precedes the occurrence of AML - it is imperative for MS patients to have an early, definitive diagnosis and to start treatment for AML as rapidly as possible. Therefore, we must cite MS as one of the differential diagnoses of cardiac tumors in any patient of leukemia. We need more prospective studies with larger patient cohorts to understand the mechanism(s) of MS development and prognosis, diagnostic approach, and treatment.

\section{Statement of Ethics}

Written informed consent has been obtained from the parents of the patient to publish the case including accompanying images

\section{Conflict of Interest Statement}

The authors have no conflict of interest to declare. 


\section{References}

1 Kashofer K, Gornicec M, Lind K, Caraffini V, Schauer S, Beham-Schmid C, et al. Detection of prognostically relevant mutations and translocations in myeloid sarcoma by next generation sequencing. Leuk Lymphoma. 2018 Feb 1;59(2):501-4.

2 Kudva R, Monappa V, Solanke G, Valiathan M, Rao ACK, Geetha V. Myeloid sarcoma: a clinicopathological study with emphasis on diagnostic difficulties. J Cancer Res Ther. 2017 Oct-Dec;13(6):989-93.

3 Wang HQ, Li J. Clinicopathological features of myeloid sarcoma: report of 39 cases and literature review. Pathol Res Pract. 2016 Sep 1;212(9):817-24.

4 Vardiman JW. The World Health Organization (WHO) classification of tumors of the hematopoietic and lymphoid tissues: an overview with emphasis on the myeloid neoplasms. Chem Biol Interact. 2010 Mar 19; 184(1-2):16-20.

5 Ohanian M, Faderl S, Ravandi F, Pemmaraju $\mathrm{N}$, Garcia-Manero G, Cortes J, et al. Is acute myeloid leukemia a liquid tumor? Int J Cancer. 2013 Aug 1;133(3):534-43.

6 Tsimberidou AM, Kantarjian HM, Wen S, Keating MJ, O’Brien S, Brandt M, et al. Myeloid sarcoma is associated with superior event-free survival and overall survival compared with acute myeloid leukemia. Cancer. 2008 Sep 15;113(6):1370-8.

7 Neiman RS, Barcos M, Berard C, Bonner H, Mann R, Rydell RE, et al. Granulocytic sarcoma: a clinicopathologic study of 61 biopsied cases. Cancer. 1981 Sep 15;48(6):1426-37.

8 Khan MY, Hussein KK, Walter MG, Hasan MK, Kern W, Kharfan-Dabaja MA. Granulocytic sarcoma presenting with malignant anasarca in a patient with secondary acute myeloid leukemia. Int J Hematol. 2004 Apr 1; 79(3):250-2.

9 Rigamonti F, Beris P, Sanchez-Pareja A, Meyer P, Ashrafpoor G, Zaza S, et al. Atypical presentation of acute myeloid leukemia: cardiac myeloid sarcoma. Int J Hematol. 2009 Jun 1; 89(5):693-8.

10 Ko YM, Lee SH, Huh J, Koo HH, Yang JH. A fatal case of acute pulmonary embolism caused by right ventricular masses of acute lymphoblastic lymphoma-leukemia in a 13 year old girl. Korean J Pediatr. 2012 Jul;55(7): 249-53.

11 Bujold KE, Dandekar S, Barbour M. Successful treatment of relapsed pediatric acute myeloid leukemia presenting as central nervous system myeloid sarcoma: a single-institution case series. J Pediatr Hematol/Oncol. 2020 May 27;42(4):319-21.

12 Tao TY, Yahyavi-Firouz-Abadi N, Singh GK, Bhalla S. Pediatric cardiac tumors: clinical and imaging features. Radiographics. 2014 Jul;34(4):1031-46.

13 Tsai J, Lee EY. MDCT imaging findings of extramedullary granulocytic sarcoma of the heart. J Thorac Imaging. 2010;25(1):W14-6.

14 Pileri SA, Ascani S, Cox MC, Campidelli C, Bacci F, Piccioli M, et al. Myeloid sarcoma: clinico-pathologic, phenotypic and cytogenetic analysis of 92 adult patients. Leukemia. 2007 Feb;21(2):340-50.

$15 \mathrm{Xu}$ LH, Wang Y, Chen ZY, Fang JP. Myeloid sarcoma is associated with poor clinical outcome in pediatric patients with acute myeloid leukemia. J Cancer Res Clin Oncol. 2020 Jan 9;146(4):1011-20.

16 Reynen K, Köckeritz U, Strasser RH. Metastases to the heart. Ann Oncol. 2004;15(3):375-81.

17 Moad JB, Jones KR. AML presenting as a mediastinal mass and right ventricular failure. Case Rep Clin Med. 2013;2.

18 Yang WC, Yao M, Chen YH, Kuo SH. Complete response of myeloid sarcoma with cardiac involvement to radiotherapy. J Thorac Dis. 2016 Jun;8(6):1323. 